\title{
Erratum to: Shipping Optimisation Systems (SOS): tramp optimisation perspective
}

Said El Noshokaty

Correspondence:

selnoshokaty@elesteshary.com

El Esteshary Information

Systems(EIS), 637 Horreya Avenue,

Genaklis, Alexandria 21411, Egypt

\section{Erratum}

Unfortunately, the original PDF version of this article [1] contained some errors. The equation $8,11,13,14,15,16,17,19,22$ all displayed incorrectly in the section of Appendix 1. The correct equations can be found below and the original article of PDF version has been updated to reflect this change.

$$
\begin{aligned}
& V_{j}^{k} \geq V_{i}^{k}-v_{i} \sum_{z \in Z^{\prime}} x_{i j z}^{k}, \quad i \in L, j \in E, \text { and } k \in S, \text { where } \sum_{z \in Z} x_{i j z}^{k}=1, \\
& V_{j}^{k} \geq V_{i}^{k}+v_{i} \sum_{z \in Z} x_{i j z}^{k}, i \in D, j \in E, \text { and } k \in S, \text { where } \sum_{z \in Z} x_{i j z}^{k}=1, \\
& W_{i}^{k} \geq W^{k}, i \in D, \text { and } k \in S, \text { where } \sum_{z=3,4} x_{i g z}^{k}=1, g \in G, \\
& V_{i}^{k} \geq V^{k}, i \in D \text {, and } k \in S \text {, where } \sum_{z=3,4} x_{i g z}^{k}=1, g \in G, \\
& N_{i}^{k} \geq N^{k}, i \in D \text {, and } k \in S \text {, where } \sum_{z=3,4} x_{i g z}^{k}=1, g \in G, \\
& W_{i}^{k} \geq W_{0}^{k}, i \in D, \text { and } k \in S, \text { where } \sum_{z=1,2} x_{i j z}^{k}=1, j \in E, \\
& T_{j}^{k} \geq T_{i}^{k}+t_{i}+\sum_{z \in Z} t_{i j z}^{k} x_{i j z}^{k}, i \in E_{f}, j \in E_{g}, \text { and } k \in S, \text { where } t_{i j z}^{k}=0, \text { and } \sum_{z \in Z} x_{i j z}^{k}=1, \\
& T_{j}^{k} \geq a_{i}, j \in E, \text { and } k \in S, \text { where } \sum_{i \in E j} \sum_{z \in Z} x_{i j z}^{k}=1, \\
& T_{g}^{k} \leq T^{k}, g \in G, k \in S, \text { where } \sum_{z=3,4} x_{i g z}^{k}=1 \text { and } i \in D,
\end{aligned}
$$

Received: 15 August 2017 Accepted: 15 August 2017

Published online: 28 August 2017

\section{Reference}

1. Said El Noshokaty (2017) Shipping Optimisation Systems (SOS): tramp optimisation perspective. J Shipp Trade 2:3. https://doi.org/10.1186/s41072-017-0021-y 